\begin{tabular}{|l|l|c|c|}
\hline & & $118-132$ & Hannover 1998 \\
\hline
\end{tabular}

\title{
Zur postglazialen Sedimentationsgeschichte des Großen Jasmunder Boddens, südliche Ostsee
}

\author{
ANNE MÜLLER*) \\ geochemical investigations, sedimentation history, sedimentation rates, Holocene, \\ Großer Jasmunder Bodden, southern Baltic Sea
}

\begin{abstract}
Kurzfassung: In der vorliegenden Arbeit wird die postglaziale Entwicklungsgeschichte des Großen Jasmunder Boddens (südliche Ostsee) anhand der Ergebnisse von stratigraphisch-geochemischen Untersuchungen an zwei Sedimentkernen aus dem Gewässer dargestellt. Die untersuchten Sedimentkerne erfassen einen Zeitraum zwischen dem Älteren Atlantikum und der Gegenwart. Der mit dem Einsetzen der Litorina-Transgression verbundene Anstieg des Meeresspiegels im Untersuchungsgebiet führte im Zentralteil des heutigen Gewässers zunächst zur Torfbildung und zur Ablagerung einer Organomudde. Diese organisch-reichen Sedimente wurden mit dem weiteren Anstieg des Meeresspiegels überflutet und von marinen Sedimenten überlagert. Die mit dem Aufbau einer Nehrung verbundene Abriegelung der Meeresbucht bewirkte im Subatlantikum eine Aussüßung des Gewässers. Die Entwicklungsgeschichte des Boddens spiegelt sich in der Geochemie der Sedimente wider. Die $\mathrm{C}_{\mathrm{org}} / \mathrm{N}$-Verhältnisse in den marin-brackischen Sedimenten deuten zusammen mit den $\delta^{13} \mathrm{C}$ - und $\delta^{15} \mathrm{~N}$-Werten auf die Dominanz autochthonen organischen Materials mit hohen Anteilen von aquatischen Makrophyten. In den rezenten Sedimenten nimmt der Planktonanteil auf Kosten der Makrophyten zu. Die $\mathrm{C}_{\text {org }} / \mathrm{S}$-Verhältnisse der Sedimente fallen in die für Süßwassermilieus typische Größenordnung. Die Pyritbildung in den Sedimenten des Großen Jasmunder Boddens ist sulfatlimitiert. Der Zusammenhang zwischen Akkumulationsraten organischen Kohlenstoffs $\left(\mathrm{C}_{\mathrm{Org}}\right.$-AR) und Sedimentakkumulationsraten (SAR) wird deutlich von der Menge, der Zusammensetzung und den ablaufenden Abbauprozessen organischer Substanz bestimmt. Erhöhte Produktivität resultiert dabei aufgrund des polymiktischen Charakters des Gewässers nicht unmittelbar in höheren Sedimentationsraten. Die Akkumulationsraten von organischem Kohlenstoff, Stickstoff und biogenem Opal nehmen ab etwa 1400 BP zur Gegenwart hin deutlich zu. Dieser Trend ist für die Akkumulationsraten von Kalziumkarbonat nicht erkennbar. Das deutet darauf hin, daß der Anteil des von autochthonen karbonatbildenden Organismen herstammenden Kalziumkarbonateintrags gegenüber allochthonen Einträgen gering ist.
\end{abstract}

*) Anschrift der Verfasserin: A. MülLER, Department of Geology, Australian National University, Canberra, ACT 0200, Australia

\section{[The postglacial sedimentation history of the Großer Jasmunder Bodden lagoon, southern Baltic Sea]}

Abstract: The postglacial sedimentation history of the Großer Jasmunder Bodden, a coastal lagoon in the southern Baltic Sea area, is discussed using the results from stratigraphical and geochemical investigations of two sediment cores. These cores cover the time period from the Older Atlantic to the present. The sea level rise, which is linked to the beginning of the LitorinaTransgression, first resulted in the formation of peat and the deposition of organic rich lacustrine mud. These organic rich sediments were then flooded by the rising sea, and marine sediments were deposited. The formation of a barrier island resulted in the separation of the Großer Jasmunder Bodden from the outer sea and in a decrease in salinity in the coastal lagoon during the Subatlantic. This development of the lagoon is reflected in the geochemical composition of the sediments. The $\mathrm{C}_{\mathrm{org}} / \mathrm{N}$ ratios in the marine-brackish sediments, together with the $\delta^{13} \mathrm{C}$ and $\delta^{15} \mathrm{~N}$ values, suggest the dominance of autochthonous organic matter including a large fraction of aquatic macrophytes. In the recent sediments there is a shift in the ratio of aquatic macrophytes and plankton to the advantage of plankton. The $\mathrm{C}_{\mathrm{org}} / \mathrm{S}$ ratios in the sediments are similiar to those of freshwater environments. Pyrite formation in the Großer Jasmunder Bodden sediments is limited by the availability of sulfate. The relationship between the accumulation rates of organic carbon $\left(\mathrm{C}_{\mathrm{org}}-\mathrm{AR}\right)$ and those of bulk sediment (SAR) is clearly determined by the amount, the composition and the prevalent decay processes of organic matter. Increased productivity however, does not directly result in higher sedimentation rates. This is due to the polymictic character of the water body. Accumulation rates of organic carbon, nitrogen and biogenic opal increase significantly after $1400 \mathrm{BP}$, though this is not the case with those of calcium carbonate. This suggests that the contribution from autochthonous carbonate forming organisms is low compared with allochthonous calcium carbonate inputs.

\section{Einleitung}

Die holozäne Entwicklungsgeschichte der Boddengewässer ist bisher nur lückenhaft stratigraphisch belegt (STRAHL 1996). Die in diesem Artikel 
dargestellten Ergebnisse der stratigraphisch-geochemischen Untersuchung von zwei ausgewählten Sedimentkernen aus dem Großen Jasmunder Bodden sollen dazu beitragen, neue Erkenntnisse zu vergangenen Entwicklungsetappen des Natursystems Boddenlandschaft zu gewinnen und frühere, vor allem auf dem Festland durchgeführte Arbeiten (z. B. KLIEWE \& JANKe 1978, 1991) zu ergänzen. Dabei wird in dieser Studie der Schwerpunkt auf geochemische Charakteristika der postglazialen Sedimente gelegt.

\section{Untersuchungsgebiet}

Der Große Jasmunder Bodden (Abb. 1) gehört zur Nordrügener Boddenkette. Letztere hat, verglichen mit den anderen Boddengewässern in der Region, die größten Ausschürftiefen und in ihrer Umrandung die höchsten Moränenhöhen (KLIEwE 1987). Der Große Jasmunder Bodden befindet sich nahezu am Ende der Nordrügener Boddenkette. Weiter „binnenwärts“ liegt nur noch der Kleine Jasmunder Bodden. Der Große Jasmunder Bodden ist in der Gegenwart ein Gewässer mit einer Fläche von $58,6 \mathrm{~km}^{2}$ und einem Volumen von $312,8 \cdot 10^{6} \mathrm{~m}^{3}$. Er weist eine mittlere Wassertiefe von 5,3 m mit einem Maximalwert von 10,3 $\mathrm{m}$ auf (CORRENS 1976).

Bei den nordrügenschen Bodden handelt es sich um ein Gewässersystem mehrerer hintereinander liegender Einzelbodden mit deutlichen Gradienten physikalischer, chemischer und biologischer Parameter. Durch Windlagen in der nördlichen Ostsee bedingte Unterschiede zwischen den Wasserständen in der südlichen Ostsee und in den Bodden führen zum Transport und lokal zur Vermischung von Wassermassen. Diese Gefälleausgleichsströmungen können auch zu einem bedeutenden Einstrom salzreicher Wassermassen führen (CORRENS \& JAEgER 1979). Der Salzgehalt in den Nordrügenschen Bodden liegt, mit Ausnahme des Kleinen Jasmunder Boddens, ganzjährig über $8 \%$, wobei die höheren Werte sowie die größere Variationsbreite erwartungsgemäß in den stärker durch die Ostsee beeinflußten äußeren Bodden zu finden sind. Der mittlere Salzgehalt schwankt zwischen 8,3 und 8,9\%o (DAHLKE \& HÜBEL 1994).

\section{Material und Methoden}

Die untersuchten Sedimentkerne GJB1 $\left(54^{\circ} 31^{\prime} 8^{\prime \prime} \mathrm{N}, 1^{\circ} 27^{\prime} 6^{\prime \prime} \mathrm{E}\right)$ und GJB2a (54032'8"N, $\left.13^{\circ} 28^{\prime} 7^{\prime \prime} \mathrm{E}\right)$ wurden im Oktober 1994 entnommen (Abb.1). Die Kerne wurden im Labor bei ihrer
Öffnung beschrieben und in $3 \mathrm{~cm}$ dicke Subproben unterteilt, wobei jede zweite Probe für Diatomeen- und Pollenanalysen verwendet wurde, deren Ergebnisse an anderer Stelle detailliert beschrieben werden (KLIBO 1995). Das übrige Material wurde gefriergetrocknet, gesiebt, vorsichtig gemörsert und homogenisiert. Die Fraktion $<0,063 \mathrm{~mm}$ wurde für die chemischen Analysen verwendet.

Der Gehalt an organischem Kohlenstoff wurde aus der Differenz der Gehalte von Gesamtkohlenstoff und anorganischem Kohlenstoff berechnet. Die Bestimmung des Gehaltes an Gesamtkohlenstoff erfolgte mit einem CHN-Analyzer (LECO CHN-1000) bei $1050{ }^{\circ} \mathrm{C}(\sigma=0,18 \%)$. Der anorganische Kohlenstoff wurde nach dem gleichem Verbrennungsprinzip bei $1200^{\circ} \mathrm{C}$ im Karbonatmodul eines C-S-Analyzers (ELTRA METALY'T CS 100) bestimmt. Zur Bestimmung des anorganischen Kohlenstoffs wurde 50\%ige Phosphorsäure zugesetzt $(\sigma=0,08 \%)$. Die Berechnung des Kalziumkarbonatgehaltes erfolgte duch Multiplikation des Gehaltes an anorganischem Kohlenstoff mit einem Faktor von 8,33.

Die Bestimmung des Gesamtstickstoffgehaltes erfolgte im gleichen Meßvorgang wie die Analyse des Gesamtkohlenstoffgehaltes $(\sigma=0,03 \%)$. Die Anwendung des $\mathrm{C}_{\text {org }} / \mathrm{N}$-Verhältnisses an Stelle des $\mathrm{C}_{\text {org }} / \mathrm{N}_{\text {ges }}$-Verhältnisses (HeBbeln 1991, RutTENBERG et al. 1997) beeinträchtigte die Dateninterpretation in dieser Studie nicht, da die Unterschiede zwischen beiden Verhältnissen gering sind (MÜLLER \& MATHESIUS 1997).

Die Bestimmung des Schwefelgehaltes erfolgte mit einem C-S-Analyzer (ELTRA METALYT CS $100)$ bei $1400^{\circ} \mathrm{C}(\sigma=0,17 \%)$.

Die Bestimmung von biogenem Opal erfolgte nach der Methode von DeMaster (1981). Dabei wurde die Mehrfachmessung durch eine Einzelmessung ersetzt. Die holozänen Sedimente sind durch eine geringe Tonfraktion und relativ hohe Gehalte von biogenem Opal gekennzeichnet. Ergebnisse von Testserien an Sedimenten der südlichen Ostsee haben gezeigt, daß die o.g. Methode die höchste Genauigkeit erreicht, wenn $\mathrm{NaOH}$ als Lösungsreagenz angewendet wird. Der biogene Opal wurde vollständig aufgelöst, und die Kontamination durch Tonminerale war minimal, wenn die Lösungszeit 40 Minuten betrug (vgl. LeIPE et al. 1995, Neumann et al. 1996). Für die Analyse wurden $100 \mathrm{mg}$ gefriergetrocknetes Probenmaterial (Fraktion < 0,063 mm) und $100 \mathrm{ml} \mathrm{1M} \mathrm{Natron-}$ lauge $(\mathrm{NaOH})$ in ein Edelstahl-Reaktorgefäß gegeben. Dieses wurde bei $85^{\circ} \mathrm{C}$ für $40 \mathrm{~min}$. in 


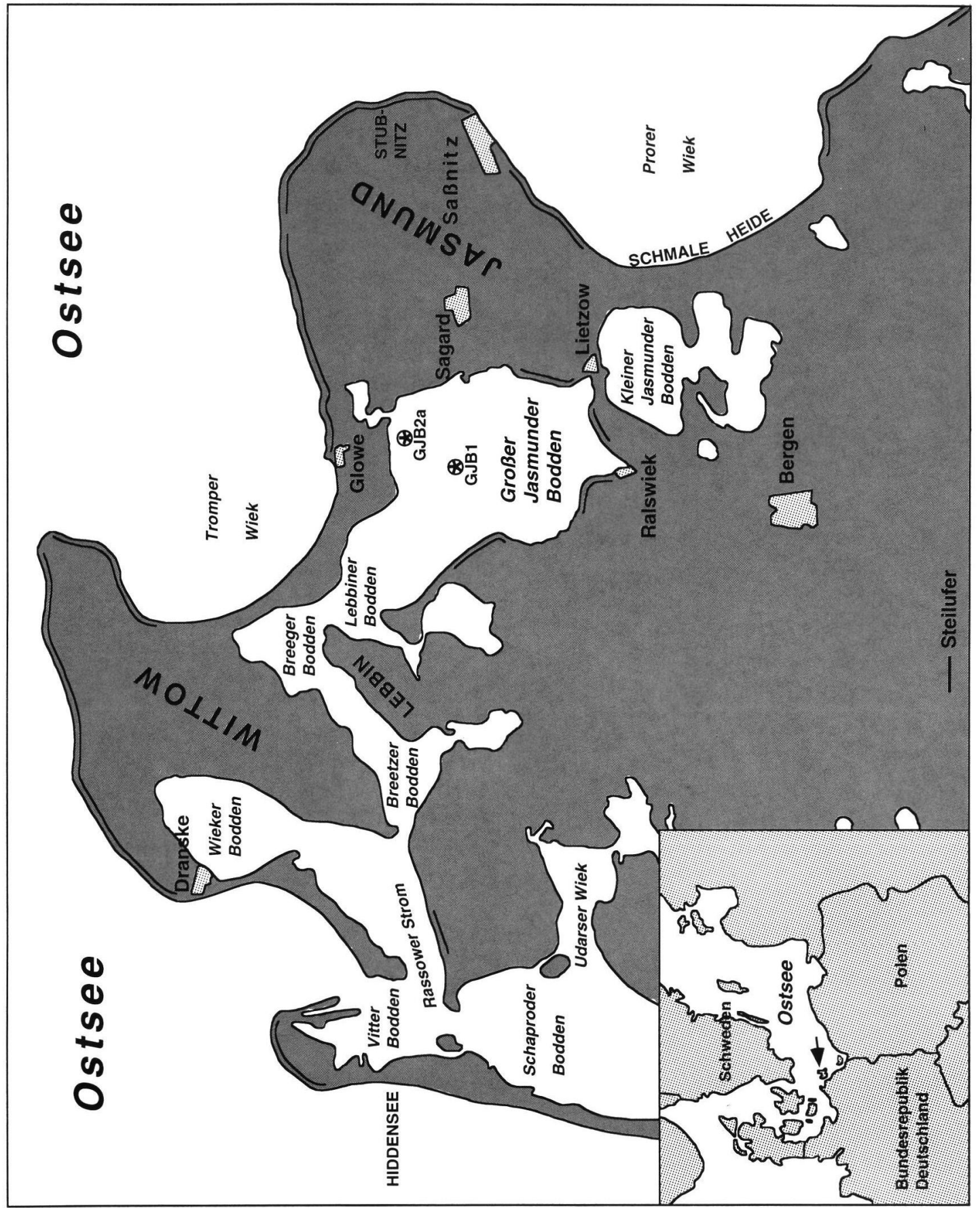

Abb. 1: Darstellung des Untersuchungsgebietes mit Beprobungspunkten.

Fig. 1: Study area and sampling locations. 
einem Wasserbad geschüttelt. Nach einer Abkühlungszeit von 2 Stunden wurde die Lösung zentrifugiert und dekantiert. Die Werte von \% Si-Opal wurden zu Gew.-\%Opal unter Berücksichtigung des gebundenen Wassergehaltes umgerechnet (Opal [Gew.- $\left.\%]=\mathrm{SiO}_{2}[\mathrm{mg} / 1] \cdot 2,4 / 10, \sigma<0,5 \%\right)$ (vgl. Mortlock \& Froelich 1989).

Für die Isotopenmessungen am organischen Material im Sediment wurde jeweils die gesamte gefriergetrocknete Probe gemörsert und in Silberschiffchen eingewogen. Die Entfernung des Karbonats erfolgte durch Zusatz von 2\%iger Salzsäure $(\mathrm{HCl})$. Das Häufigkeitsverhältnis der stabilen Kohlenstoff-Isotope ${ }^{12} \mathrm{C}$ und ${ }^{13} \mathrm{C}$ und der stabilen Stickstoff-Isotope $14 \mathrm{~N}$ und $15 \mathrm{~N}$ wurde an reinem $\mathrm{CO}_{2}$ - bzw. $\mathrm{N}_{2}$-Gas mit einem Finnigan MAT Delta S-Massenspektrometer bestimmt, das an einen Carlo Erba/Fisons 1108 CHN-Analyzer gekoppelt war $\left(\sigma=0,15 \%\right.$ für $\delta^{13} \mathrm{C}$ bzw. $<0,2 \%$ für $\left.\delta^{15} \mathrm{~N}\right)$. Als Referenzgas diente reines $\mathrm{CO}_{2} \mathrm{bzw} . \mathrm{N}_{2}$, das gegen Karbonat (NBS-18, -19 bzw. -20) bzw. atmosphärischen Stickstoff (MARIOTTI 1983) als Standard kalibriert war. Die folgenden Formeln (FAURE 1986) wurden zur Berechnung angewendet:

$$
\left.\delta 13 \mathrm{C}(\% 0)=\left[\frac{(13 \mathrm{C} / 12 \mathrm{C})_{\text {Probe }}}{(13 \mathrm{C} / 12 \mathrm{C})_{\text {Referenz }}}-1\right)\right] \cdot 1000
$$

und

$$
\left.\delta 15 \mathrm{~N}(\% 0)=\left[\frac{(15 \mathrm{~N} / 14 \mathrm{~N})_{\text {Probe }}}{(15 \mathrm{~N} / 14 \mathrm{~N})_{\text {Referenz }}}-1\right)\right] \cdot 1000
$$

Die Sedimente wurden mit Hilfe von Pollenanalysen datiert (KLIBO 1995). Ein Pollenprofil für das Oderhaff, das die einzelnen Pollenzonen zeigt, wurde in MüLLER et al. (1996) veröffentlicht. Eine detaillierte Darstellung eines Pollenprofils aus der Region des Greifswalder Boddens findet sich zudem in Strahl (1996), wobei die Ergebnisse mit neueren Studien auf der Insel Rügen (z. B. LANGE et al. 1986) verglichen werden. In den Schlicken wird eine tiefenbezogene Altersansprache oft durch die vorhandene Sedimentdurchmischung erschwert. Trotz dieser abschnittsweise auftretenden Schwierigkeiten sind die zur zeitlichen Datierung erforderlichen pollenanalytischen Kennwerte, wie z. B. Buchenmaximum, Getreide- und Kulturbegleiterkurve, Linden- und Ulmenmaximum, gut ausgeprägt (MülLER et al. 1996). Für eine ausführliche Diskussion zur Entwicklung eines regionalen Altersschemas mit Hilfe der Pollenanalyse und ${ }^{14} \mathrm{C}$-Datierungen sowie möglicher Fehlerquellen sei zudem auf die Arbeit von KuIEwE \& JANKE (1982) verwiesen. Basierend auf den Ergebnissen der Pollenanalyse des Kerns GJB1 wurden lineare Sedimentationsraten berechnet.

Zur unabhängigen Quantifizierung des Eintrages von Kalziumkarbonat, organischem Kohlenstoff und biogenem Opal von dem terrigenen Eintrag und anderen biogenen Komponenten wurden die Gewichtsprozentanteile in Akkumulationsraten umgerechnet. Die Akkumulationsraten des Gesamtsediments $\left(\mathrm{AR}_{\text {ges }}\right)$ und der biogenen Komponenten $\left(\mathrm{AR}_{\mathrm{komp}}\right)$ wurden auf der Grundlage der oben erwähnten linearen Sedimentationsraten nach VAN ANDEL et al. (1975) und ThIEDE et al. (1982) berechnet und in $\mathrm{g} /\left(\mathrm{m}^{2} \cdot \mathrm{Jahr}\right)$ angegeben (MÜller \& Suess 1979, ReA et al. 1980). Zur Bestimmung des Trockenraumgewichts wurde bei Öffnung der Kerne für jedes 3-cm-Intervall mit einem Stechzylinder ein gegebenes Volumen an Sediment entnommen, das bei $60^{\circ} \mathrm{C}$ im Trockenschrank bis zur Gewichtskonstanz getrocknet und dann gewogen wurde.

\section{Ergebnisse und Diskussion}

\subsection{Sedimentationsgeschichte}

Im Großen Jasmunder Bodden wurde eine Sedimentabfolge beginnend ab dem Älteren Atlantikum erfaßt. In diesem Zeitabschnitt erreichte das Litorina-Meer das Gebiet der heutigen südlichen Ostseeküste. Der ansteigende Meeresspiegel führte zunächst zur Vernässung, mit der im Großen Jasmunder Bodden ein Torfwachstum und die Ablagerung von Organomudde verbunden waren. So liegt im zentralen Teil des Gewässers (Kern GJB 1) unter dem bei 5,45 m (-11,20 m $\mathrm{NN}$ ) einsetzenden Schlick ein organisch-reiches Sediment in Form von Organomudde bis Schilftorf an, das im weiteren Verlauf dieser Studie bei der Diskussion der geochemischen Ergebnisse vereinfacht als Organomudde bezeichnet werden soll. Diese organisch-reichen Ablagerungen wurden durch den schnellen Anstieg des Meeresspiegels überflutet und es wurden marine Sedimente abgesetzt. Die einzelnen Höhepunkte der Litorina-Transgression lassen sich in den Sedimenten des Großen Jasmunder Boddens mit Hilfe geochemischer Methoden nicht nachweisen. Die gegenwärtige, auf Untersuchungen auf dem Festland basierende Arbeitshypothese im Rahmen des Projektes „Klimawirkung und Boddenlandschaft" besagt, daß zum Litorina-Meer eine weitgehend offene Verbindung in Form einer tief in das Binnenland hineinreichenden Meeresbucht bestand. Im Verlauf des Subatlantikums erfolgte die Verbindung der seeseitigen Strandwall- 
und Dünensysteme zu einer weitgehend geschlossenen Nehrung durch fortwährenden Küstenausgleich. Die Folgen waren die Abriegelung der Meeresbucht und eine damit verbundene Aussüßung des nun landseitigen Wasserbeckens (KLIBO 1995). Im nordöstlichen Teil des Großen Jasmunder Boddens (Kern GJB2a), d. h. in unmittelbarer Nähe der Schaabe, der heutigen Nehrung (vgl. Abb. 1), wurden dagegen bis in etwa $0,75 \mathrm{~m}$ Tiefe Mittelsande mit organisch-reichen Einschlüssen erbohrt.

\subsection{Zusammensetzung der organischen Substanz in den Sedimenten}

Bei sedimentologischen Arbeiten im ozeanischen Bereich hat man sich des $\mathrm{C} / \mathrm{N}$-Verhältnisses zur Unterscheidung organischer Substanz mariner/ autochthoner bzw. terrestrischer Herkunft bedient (z. B. Hebbeln 1991, Ruttenberg \& GoÑI 1997). So gehen BordovskIY (1965) von einem $\mathrm{C} / \mathrm{N}-$ Verhältnis von $\leq 8$ in mariner und PRAHL et al. (1980) von $>12$ in terrigener organischer Substanz aus. Das $\mathrm{C} / \mathrm{N}-$ Verhältnis findet bei dieser Unterscheidung Anwendung, da es den Anteil von Eiweißen, den wichtigsten stickstoffhaltigen Substanzen in Organismen ausdrückt (MüLLER 1975). C/N-Verhältnisse von Plankton mit relativ hohen Eiweißanteilen z. B. liegen in der Regel zwischen 4 und 7 (BordovskIY 1965), während Landpflanzen mit Eiweißanteilen von nur 1-10\% $\mathrm{C} / \mathrm{N}-$ Verhältnisse von 30 und mehr erreichen können (NAKAI 1986). Für die Bodden und Haffe kann darüber hinaus angenommen werden, daß Makrophyten, die das Ufer besiedeln, erheblich zum Eintrag organischer Substanz in das Sediment beitragen. Für aquatische Makrophyten in den Boddengewässern geben CONRAD et al. (1995) ein mittleres C/N-Verhältnis von 13,6 an. Zudem können Erosionsprozesse von Torf und Böden, die Reste von Landpflanzen enthalten, für die Bodden und Haffe der südlichen Ostsee eine nicht unerhebliche Rolle beim Eintrag organischer Substanz in das Sediment spielen.

In den Sedimenten des Großen Jasmunder Boddens (Kern GJB1) liegen die $\mathrm{C}_{\text {org }}$ / N-Verhältnisse im Älteren und Jüngeren Atlantikum zwischen 8 und 12 (Abb. 2). Zusammen mit den Isotopenwerten $(\delta 13 \mathrm{C}, \delta 15 \mathrm{~N})$ deuten diese Verhältnisse auf die Dominanz autochthoner organischer Substanz mit einem hohem Beitrag von aquatischen Makrophyten. Die Verbindung zum Litorina-Meer wurde in der Folgezeit durch das Wachstum von Haken eingeengt, das an der vorpommerschen

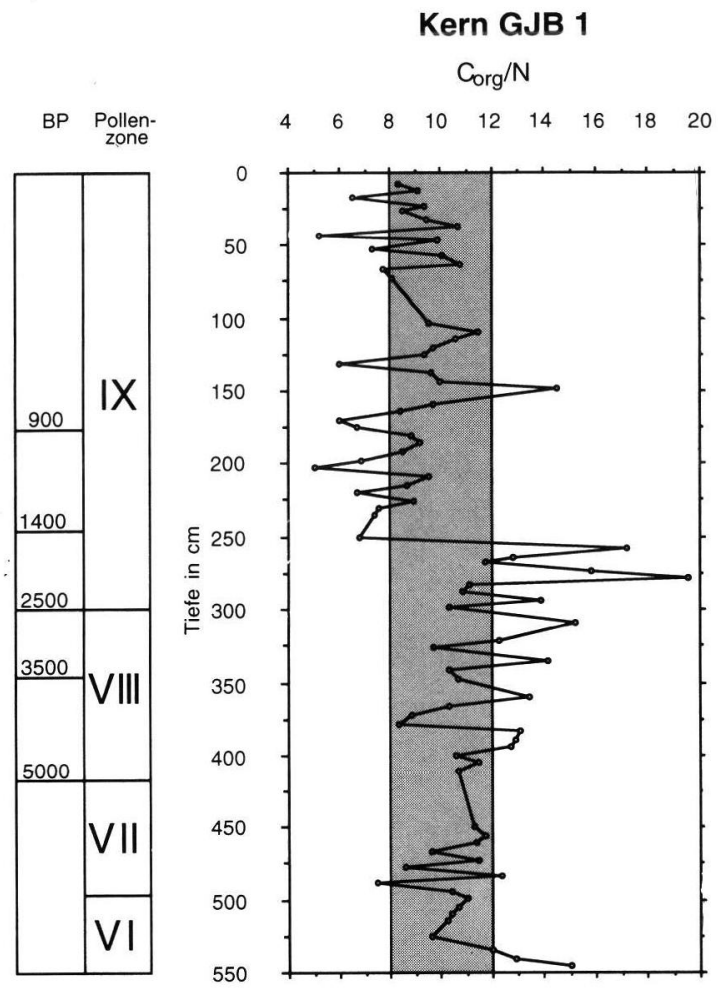

Abb. 2: Darstellung der $\mathrm{C}_{\mathrm{org}} / \mathrm{N}$-Verhältnisse (von Gewichtsprozenten) gegen die Tiefe für den Sedimentkern GJB1. Verhältnisse größer als 20 sind nicht dargestellt. Am linken Rand sind die zeitliche Einordnung BP und Pollenzonen angegeben. Das schattierte Feld markiert die typischen Endwerte für $\mathrm{C} / \mathrm{N}$-Verhältnisse mariner bzw. terrigener organischer Substanz nach BORDOVSKIY (1965) bzw. Prahl et al. (1980).

Fig. 2: Plot of $\mathrm{C}_{\text {org }} / \mathrm{N}$ ratios (of weight $\%$ ) versus depth for sediment core GJB1. Ratios larger than 20 are not shown. On the left margin, years BP and pollen zones are illustrated. The shaded area marks the typical endpoints for $\mathrm{C} / \mathrm{N}$ ratios of marine and terrigenous organic matter according to BORDOVSKIY (1965) and PraHL et al. (1980) respectively.

Küste hauptsächlich im Subboreal begann (KuIEWE \& JANKE 1991). Vom Subboreal bis zum Beginn des Subatlantikums steigen die $\mathrm{C}_{\text {org }} / \mathrm{N}$-Werte. deutlich an, wobei sie bis 20 erreichen können. Das deutet darauf hin, daß das Phytoplankton in dieser Zeit relativ zurückgedrängt wurde.

Die vollständige Abriegelung des Großen Jasmunder Boddens von der offenen See erfolgte wahrscheinlich im Subatlantikum (KuıBO 1995). Stärkere Rodungen fanden im Gebiet um den Großen Jasmunder Bodden schon ab 1400 BP statt (LANGE et al. 1986). Es liegt nahe, daß es 


$$
\delta^{13} \mathrm{C}(\% \circ)
$$

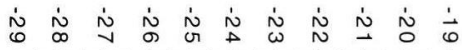

\section{Kern GJB1}
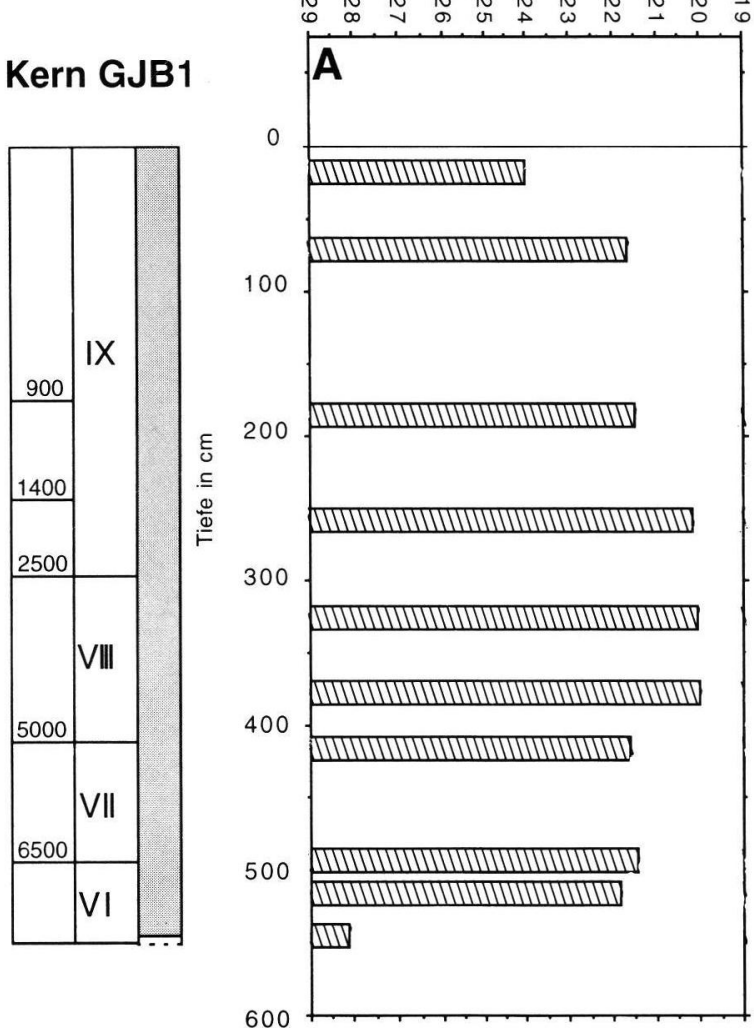

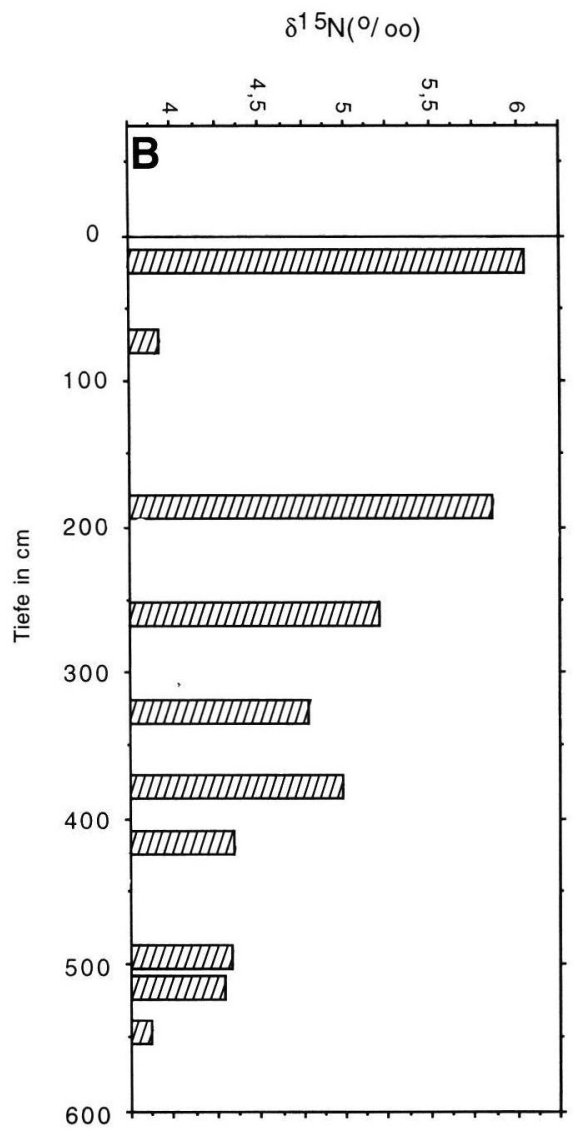

Abb. 3: Verhältnisse stabiler Isotope in den Sedimenten des Großen Jasmunder Boddens (Kern GJB1): (A) organischer Kohlenstoff, $\delta^{13} \mathrm{C}$, und (B) Stickstoff $\delta^{15} \mathrm{~N}$. Links: Pollenzonen mit Altersangaben (BP und Sedimentabfolge. Letztere zeigt Schlicke (grau) und Organomudde an der Basis (dunkel). Isotopendaten aus MüLLER \& Voss (1997).

Fig. 3: Stable isotope ratios of (A) organic carbon, $\delta^{13} \mathrm{C}$, and (B) nitrogen, $\delta^{15} \mathrm{~N}$, for the Großer Jasmunder Bodden sediment core GJB1. The three left columns show years BP, pollen zones and the sediment sequence. The sediment sequence from the top shows mud (gray) and organic rich lacustrine mud (dark). The isotope data is from MÜlLER \& VOSS (1997).

durch diese Faktoren zu einer erhöhten Nährstoffzufuhr in den Bodden und zu einem Fortschreiten der Eutrophierung kam, wobei Einträge aufgrund des relativ ausgeprägten Reliefs um den Bodden sicherlich noch verstärkt auftreten konnten. Ab etwa 1400 BP sind die $\mathrm{C}_{\text {org }}$ /N-Verhältnisse in den Sedimenten deutlich niedriger, wobei sie zeitweise sogar in den Bereich fallen, der für Plankton typisch ist. In den letzten 350-400 Jahren streuen die C/N-Verhältnisse zwischen 6 und 11, mit der Mehrzahl der Werte zwischen 8 und 10. Diese Zahlen legen einen hohen Anteil autochthoner organischer Substanz nahe.

Die Oberflächensedimente des Kerns GJB 2a aus dem Großen Jasmunder Bodden haben im Ver- gleich zu den obersten $50 \mathrm{~cm}$ des Kerns GJB1 höhere $\mathrm{C}_{\text {org }} / \mathrm{N}-$ Verhältnisse, die alle $>10$, größtenteils sogar $>12$ sind. Neben den $\mathrm{C} / \mathrm{N}$-Verhältnissen sind auch die stabilen Isopenverhältnisse organischer Substanz $\left(\delta^{13} \mathrm{C}, \delta^{15} \mathrm{~N}\right)$ benutzt worden, um die Herkunftsquellen des in das Sediment eingelagerten organischen Materials zu bestimmen und um Informationen zu den Sedimentationsvorgängen abzuleiten (z. B. THORNTON \& McManus 1994). In gemäßigten Breiten werden $\delta 13 \mathrm{C}$-Werte zwischen $-25 \%$ bis $-30 \%$ für den überwiegenden Teil (d. h. von C3-Pflanzen herstammend) der terrigenen organischen Substanz angegeben (Fontugne \& JouannEau 1987, EMERSON \& HEDGES 1988). Im marinen Milieu variieren $\delta 13 \mathrm{C}$-Werte zwischen - $19 \% 0$ und -22\%o in Anhän- 
gigkeit vom hydrologischen Regime1) (FONTUGNE \& JOUANNEAU 1987). Für Süßwasserplankton wird davon ausgegangen, daß dieses niedrigere $\delta^{13} \mathrm{C}$ Werte hat als marines Plankton, wobei GaLimow (1985) einen Schwankungsbereich von $-20 \%$ bis $-30 \%$ angibt.

Die $\delta 15 \mathrm{~N}$-Werte für terrestrische Pflanzen bewegen sich zwischen $-5 \%$ und $+18 \%$, sind aber im Durchschnitt $(+3 \%)$ isotopisch leichter. Stickstoffixierende Pflanzen haben einen Bereich von $-6 \%$ bis $+6 \%$ (Schoeninger \& DeNiro 1984). Marine Organismen haben mittlere $\delta 15 \mathrm{~N}$-Werte zwischen +7 und $+10 \%$ (PETERS et al. 1978). $\delta 15 \mathrm{~N}$ Signaturen sind deutlichen Modifikationen durch Isotopenfraktionierung ausgesetzt, die während der biogenen Umformung und im Kreislauf der gelösten und partikulären Stickstoffverbindungen auftritt. Biogeochemische Hauptprozesse, die die $\delta 15 \mathrm{~N}-Z$ Zusammensetzungen der verschiedenen Stickstofformen modifizieren, schließen Ammonifizierung, Nitrifizierung, Denitrifikation, dissimilative Nitratreduktion und Nährstoffassimilation ein, wobei die Isotopenfraktionierung bei jedem dieser Prozesse bewirkt, daß die Reaktionsprodukte isotopisch leichter sind als die Ausgangssubstrate (Owens 1987). Der allgemeine Effekt der Diagenese organischer Substanz ist folglich, daß der residuale Stickstoff, durch die Anreicherung von $15 \mathrm{~N}$, vom Charakter her "mariner" wird, d. h. daß die Sedimente scheinbar einen höheren Anteil autochthonen Materials aufweisen. Dieser Effekt tritt ungeachtet der Herkunft von frischem organischen Material auf und hat damit ernsthafte Auswirkungen für Isotopenstudien, denn der gemessene Wert kann so das Ausmaß der biogenen Veränderungen unter Umständen deutlicher reflektieren als die Herkunft des Materials (SWEENEY \& KaPLAN 1980, THORNTON \& McManus 1994).

Die Isotopenverhältnisse aquatischer Makrophyten zeigen in den Bodden einen breiten Schwankungsbereich zwischen $-11,4$ und $-28,1 \%$ o für $\delta^{13} \mathrm{C}$ und zwischen 9,5 und $14,2 \%$ für $\delta^{15} \mathrm{~N}$. Planktonproben aus dem Kleinen Haff und aus dem Greifswalder Bodden haben niedrige $\delta 13 \mathrm{C}$ Werte zwischen $-24,3 \%$ und $-25,1 \%$ und ent-

1) Unter marinen Bedingungen werden hier voll-saline Milieus im Weltmeer verstanden, die eine Salinität von etwa 30 bis 35\% aufweisen (TOMCZAK \& GODFrEY 1994). Der nachfolgend in dieser Arbeit verwendete Begriff "backisch" bezieht sich auf die typischen Salinitäten der südlichen Küstengewässer der Ostsee von in der Regel < 10\%0 (BIRR \& OERTEL 1993 und Referenzen darin). sprechende $\delta 15 \mathrm{~N}-W e r t e$ zwischen $12,5 \%$ und $15,5 \%$ (MülLER \& Voss 1997). Ausgedehnte Studien der $\delta 15 \mathrm{~N}-$ Verhältnisse des Planktons in den brackischen Boddengewässern der vorpommerschen Küste liegen bisher noch nicht vor. Erste Ergebnisse aus dem Oderhaff deuten jedoch darauf hin, daß die $\delta 15 \mathrm{~N}-W e r t e$ im $\mathrm{NO}_{3}$ und damit in partikulärer organischer Substanz aufgrund erhöhter Nährstoffkonzentration und unvollständiger Aufnahme von $\mathrm{NO}_{3}$ höher sind als die zuvor angegebenen Durchschnittswerte aus dem marinen Bereich (VOSS \& STRUCK 1997).

Die Kohlenstoffisotopenwerte im Sedimentkern GJB1 unterstützen den Trend der C/N-Verhältnisse (Abb. 3 A). Die an der Basis des Sedimentkerns in $5,45 \mathrm{~m}$ Tiefe $(-11,20 \mathrm{~m} \mathrm{NN}$, Älteres Atlantikum) erbohrte Organomudde zeigt einen $\delta 13 \mathrm{C}-$ Wert von $-28,1 \%$, der typisch für terrestrische C3Pflanzen ist. Dieser niedrige Wert ist auf die Schilftorfanteile in dem organisch-reichen Sediment zurückzuführen. Die Schlickproben aus dem Älteren und Jüngeren Atlantikum haben $\delta^{13} \mathrm{C}$-Werte zwischen $-21,8 \%$ und $-21,4 \%$. Zusammen mit den $\mathrm{C} / \mathrm{N}-$ Verhältnissen belegen diese Werte die Einlagerung autochthoner organischer Subtanz in den Sedimenten, wobei die Makrophyten gegenüber dem Plankton überwogen haben müssen.

Im Subboreal und zu Beginn des Subatlantikums nehmen die Kohlenstoffisotopenwerte ab (- 20,2 bis - 20,0\%), steigen aber um 1000 BP wieder leicht an $(-21,5 \%)$. Um ca. 400 BP liegt ein Wert von $-21,6 \%$ vor. In den Oberflächensedimenten wurden $-24,0 \%$ gemessen. Die Kohlenstoffisotopenwerte zeigen in Übereinstimmung mit den $\mathrm{C}_{\text {org }} / \mathrm{N}$-Werten, daß im Subboreal und zu Beginn des Subatlantikums (bis ca. 1500 BP) hauptsächlich autochthone organische Substanz mit einem hohen Anteil aquatischer Makrophyten in das Sediment eingelagert wurde. Für den Zeitraum zwischen 900 und 400 BP liegt keine Isotopenmessung vor, die $\mathrm{C}_{\text {org }} / \mathrm{N}$-Werte deuten jedoch darauf hin, daß in diesem Zeitraum neben aquatischen Makrophyten große Anteile von Plankton eingetragen wurden. Der Isotopenwert um 400 BP zeigt deutlich, daß hauptsächlich autochthone organische Substanz in Form von Plankton (und möglicherweise auch aquatischen Makrophyten) in das Sediment eingelagert wurde. Der deutlich höhere Kohlenstoffisotopenwert in den rezenten Sedimenten kann nur durch eine Zunahme des Planktonanteils auf Kosten der Makrophyten bedingt sein. 
Die $\delta^{15} \mathrm{~N}$-Werte unterstützen die oben gegebene Interpretation (Abb. $3 \mathrm{~B}$ ). Die Organomudde hat einen $\delta 15 \mathrm{~N}$-Wert von 3,9\%o, der auf terrestrischen Eintrag hindeutet. Die darüberliegenden Schlickproben haben Werte von jeweils 4,3\%o im Älteren und Jüngeren Subalantikum. Ab dem Subboreal $(4,4 \% 0)$ ist ein zunehmender Trend der $\delta 15 \mathrm{~N}$-Werte bis zu $6 \%$ an der Sedimentoberfläche zu verzeichnen. Ein solcher Trend würde einen zunehmend autochthonen Charakter des organischen Materials in den Sedimenten bedeuten. Setzt man den $\delta^{15} \mathrm{C}$-Wert in den Oberflächensedimenten in Bezug zu dem $\delta 13 \mathrm{C}$-Wert der gleichen Probe, so kann der Stickstoffisotopenwert einerseits durch einen erhöhten Anteil autochthonen organischen Materials, insbesondere Plankton, bedingt sein. Andererseits ist auch nicht auszuschließen, daß wie im Oderhaff - erhöhte Nährstoffkonzentration und unvollständige Aufnahme von $\mathrm{NO}_{3}$ den höheren $\delta 15 \mathrm{~N}-$ Wert verursacht hat (MüLLER \& Voss 1997).

\subsection{Kohlenstoff-Schwefel-Systematik in den Sedimenten}

Eine Darstellung der Gehalte an Schwefel gegen organischen Kohlenstoff unter Anwendung des empirischen Diagramms von BERNER \& RAISWELL (1983) wurde benutzt, um das Paläomilieu des Großen Jasmunder Boddens zu charakterisieren (Abb. 4). Das markierte Feld um die Regressionsgerade kennzeichnet die typischen Werte für normale Meeressedimente mit oxischem Bodenwasser. Die Begriffe „anoxisch“, „normal marin/oxisch" und "nicht-marin“ dienen dazu, das bodennahe Wasser zur Zeit der Ablagerung zu charakterisieren (EMEIS \& MORSE 1993). Von den Sedimenten aus dem Großen Jasmunder Bodden (Kerne GJB1 und GJB2) liegen einige wenige Datenpunkte in dem Bereich, der für normale Meeressedimente definiert ist (Abb. 4). Die Mehrzahl der Proben fällt in das für Süßwasserbedingungen typische Feld. In dem hier betrachteten Gewässer korrelieren die $\mathrm{C}_{\text {org }}$-Gehalte in

\section{Kerne GJB1 und GJB2a, $r=0.71$}

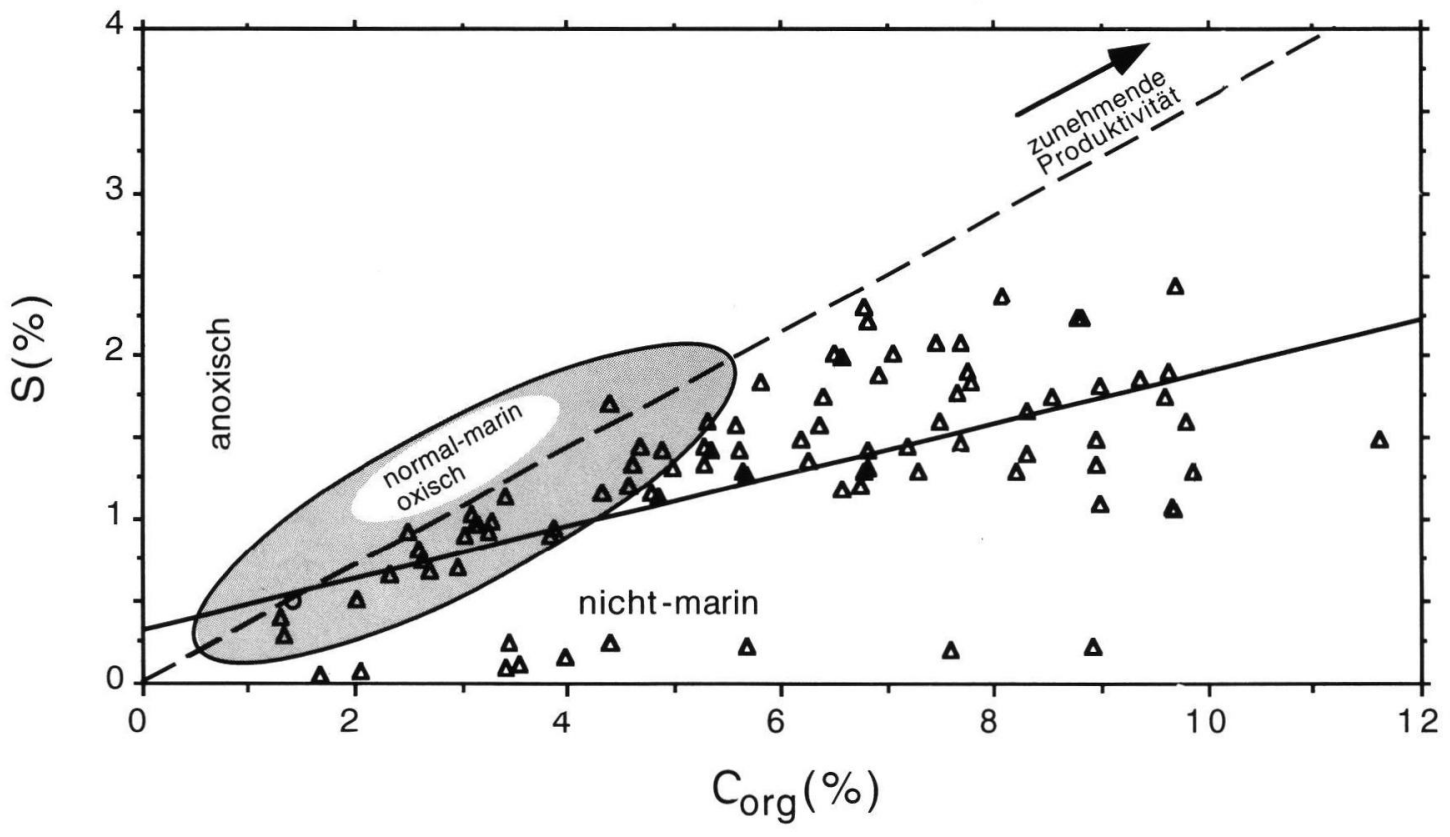

Abb. 4: Darstellung der Gehalte an organischem Kohlenstoff und Schwefel (jeweils in Gewichtsprozent) in den Sedimenten des Großen Jasmunder Boddens (Kerne GJB1 und GJB2a). Der schattierte Bereich markiert das empirisch bestimmte Feld für normale Meeressedimente nach BERNER \& RAISWELL (1983). Die unterbrochene Linie im Diagramm ist die Regressionsgerade zu den Datenpunkten der o. g. Autoren, die durchgehende Linie ist die Regressionsgerade zu den hier gezeigten Daten.

Fig. 4: Plot of sulfur versus organic carbon content (both in weight \%) for the Großer Jasmunder Bodden samples (cores GJB 1 and GJB 2a). The shaded area marks the empirically defined region for normal marine sediments according to Berner \& Raiswell (1983). The dashed line represents a best fit to the data in their study. The solid line represents a best fit to the data in this study. 
den untersuchten Sedimenten positiv mit den Schwefelgehalten $(r=0,71, p<0,05)$, wobei sie einen deutlich geringeren Anstieg aufweisen als die der normalen marinen Sedimente nach BERNER \& RAISWELL (1983).

Bis etwa $1500 \mathrm{BP}$ sind überwiegend $\mathrm{C}_{\text {org }} / \mathrm{S}$-Verhältnisse zwischen 2,8 und $5 \mathrm{zu}$ beobachten (Abb. 5). Diese Werte liegen im Grenzbereich zwischen normal marinen bis limnischen Bedingungen und unterstützen die Hypothese, daß Verhältnisse dieser Größenordnung typisch für die hier untersuchten brackischen Flachwassermilieus der Bodden sind. Ähnliche $\mathrm{C}_{\text {org }} / \mathrm{S}-\mathrm{Ver}-$

\section{Kern GJB1}

$$
\mathrm{C}_{\text {org }} / \mathrm{S}
$$

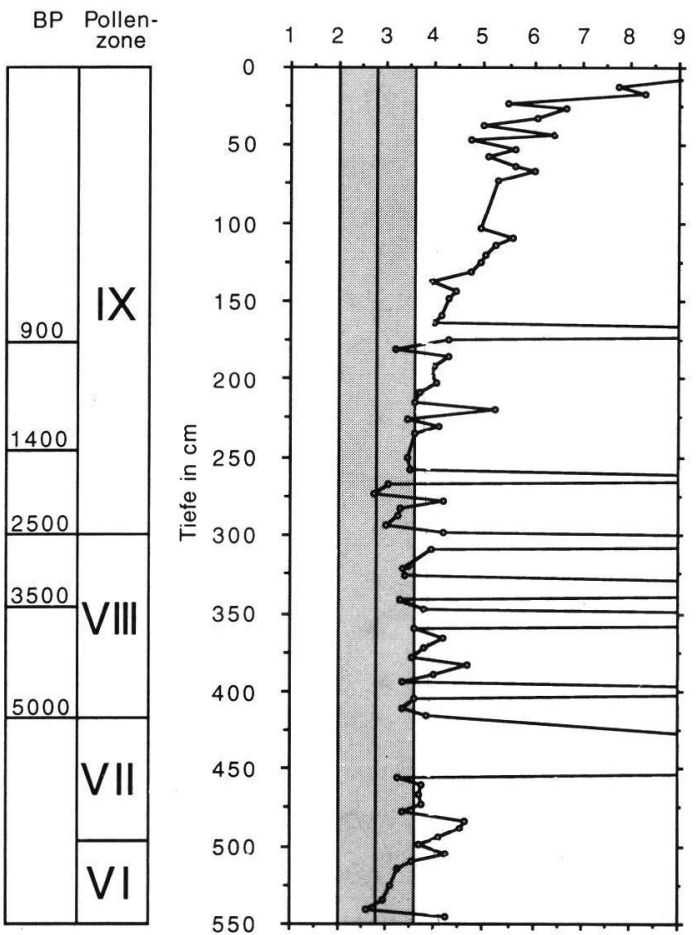

Abb. 5: Darstellung der $\mathrm{C}_{\text {org }} / \mathrm{S}$-Verhältnisse (von Gewichtsprozenten) gegen die Tiefe für die Sedimentproben des Kerns GJB1. Verhältnisse größer als 9 sind nicht dargestellt. Am linken Rand sind die zeitliche Einordnung BP und Pollenzonen angebeben. Der markierte Bereich ist typisch für normale Meeressedimente (BERNER \& RAISWELL 1983).

Fig. 5: Plot of $\mathrm{C}_{\text {org }} / \mathrm{S}$ ratios (of weight \%) versus depht for sediment core GJB1. Ratios larger than 9 are not shown. On the left margin, years BP and pollen zones are illustrated. The shaded area marks the region which is typical for normal marine sediments (BERNER \& RAISWELL 1983). hältnisse in den Sedimenten wurden bereits für den nördlichen Barther Bodden beschrieben (MüLlER 1997). Zusätzlich treten im Kern GJB1 immer wieder einzelne Lagen mit hohen C/S-Verhältnissen > 9 auf. Ab ca. 1500 BP nehmen die $\mathrm{C}_{\text {org }}$ /S-Verhältnisse kontinuierlich bis auf Werte um 9 in den rezenten Sedimenten nahe der Oberfläche zu. Die mit der Abriegelung der Meeresbucht verbundene Aussüßung des landseitigen Wasserbeckens im Subatlantikum führte offensichtlich zu höheren C/S-Verhältnissen in den Sedimenten. Die Diatomeenflora in diesem Abschnitt des Subatlantikums deutet ebenfalls auf eine Aussüßung des Gewässers hin (JANKE, pers. Mitt., KLIBO 1995). Die höheren $\mathrm{C}_{\mathrm{org}} / \mathrm{S}$-Verhältnisse im oberen Abschnitt des Sedimentkerns können jedoch zumindest teilweise auch dadurch bedingt sein, daß die frühdiagenetische Pyritbildung noch nicht abgeschlossen ist.

Im Rahmen dieser Arbeit erfolgte keine Berechnung des Pyritisierungsgrades und keine Messung der Porenwasser-Sulfatkonzentrationen. Daher müssen Aussagen allein unter Anwendung der herkömmlichen Klassifizierung nach BERNER (1970) gegeben werden. Unter der Berücksichti-

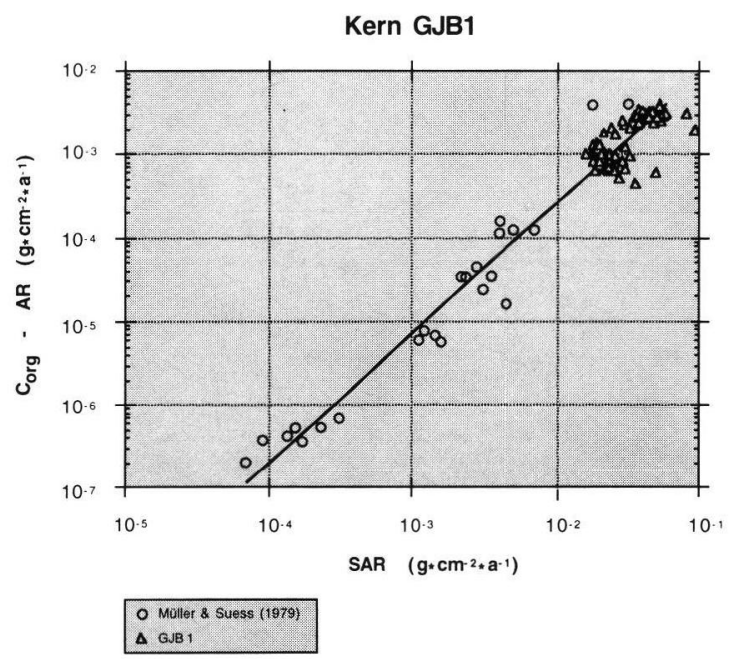

Abb. 6: Logarithmische Darstellung der Akkumulationsraten des Gesamtsediments (SAR) und des organischen Kohlenstoffs ( $\mathrm{C}_{\text {org }}-\mathrm{AR}$ ) für die Sedimente aus dem Großen Jasmunder Bodden (Kern GJB1) im Vergleich mit den Daten und der Regressionsgerade aus MülLER \& SuEss (1979).

Fig. 6: Logarithmic plot of bulk sediment accumulation rates (SAR) versus accumulation rates of organic carbon $\left(\mathrm{C}_{\mathrm{Org}}-\mathrm{AR}\right)$ for the Großer Jasmunder Bodden sediments (core GJB1). The data and the regression line from MülLER \& Suess (1979) are shown for comparison. 
gung der bekannten Parameter sollte jedoch eine allgemeine Einschätzung möglich sein. Man kann davon ausgehen, daß die Pyritbildung im Großen Jasmunder Bodden - wie auch generell unter Süßwasserbedingungen - sulfatlimitert ist. Aufgrund geringer Salinität gehen die Sulfatreduktion und die Pyritbildung in den Sedimenten mit geringerer Intensität vonstatten als bei normalen Meeressedimenten mit gleich hohen Anteilen an organischem Kohlenstoff.

Abb. 6 zeigt eine logarithmische Darstellung der Akkumulationsraten organischer Substanz $\left(\mathrm{C}_{\text {org }}{ }^{-}\right.$ AR) gegen Sedimentakkumulationsraten (SAR) für die Proben des Sedimentkerns GJB1. Zum Vergleich sind in dem Diagramm auch die Datenpunkte mit der empirischen Regressionsgerade von Müller \& Suess (1979) dargestellt, die von

\section{(1) $-y=0.0099789+0.0052586 \log (x) R=0.81591$}

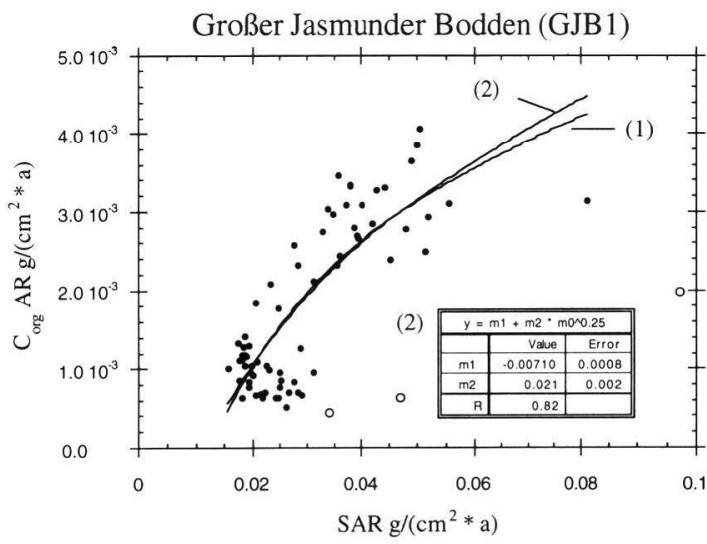

Abb. 7: Darstellung der Akkumulationsraten von organischem Kohlenstoff gegen die Sedimentakkumulationsraten (jeweils in $\mathrm{g} \cdot \mathrm{m}^{-2} \cdot \mathrm{a}^{-1}$ ) für die Sedimente aus dem Großen Jasmunder Bodden (Kern GJB1).

Fig. 7: Plot of organic carbon accumulation rates versus sediment accumulation rates (both in $\left.\mathrm{g} \cdot \mathrm{m}^{-2} \cdot \mathrm{a}^{-1}\right)$ for the Großer Jasmunder Bodden sediments (core GJB1).

einer größeren Menge von Oberflächenproben aus verschiedenen marinen Milieus herstammen. Die Sedimente aus dem Großen Jasmunder Bodden liegen um die Regressionsgerade und zeigen somit den von MülLer \& Suess (1979) beschriebenen Zusammenhang. Die Sedimentakkumulationsraten sind jedoch für die Proben aus dem Großen Jasmunder Bodden deutlich höher als für die meisten der von MülLER \& Suess (1979) gezeigten marinen Sedimente.

Eine Darstellung der $\mathrm{C}_{\text {org }}$-Akkumulationsraten gegen die Sedimentakkumulationsraten (Abb. 7) zeigt, daß bei niedrigen Sedimentakkumulationsraten zunächst ein steiler Anstieg der $\mathrm{C}_{\text {org }}$-Akkumulationsraten vorliegt. Mit wachsenden Sedimentakkumulationsraten nehmen die $\mathrm{C}_{\text {org }}$-Akkumulationsraten jedoch nicht mehr in gleichem Maße zu wie die Sedimentakkumulationsraten. Geht man von einem potenziellen Zusammenhang zwischen beiden Parametern aus, so wird die Steilheit der entsprechenden Anpassungskurve (2) durch einen Wert des Faktors $\mathrm{m}^{2}$ von 0,001 ausgedrückt. In Abbildung 7 wird aber ebenfalls deutlich, daß eine andere Art der Anpassung, z. B. in logarithmischer Form, nicht auszuschließen ist (Kurve 1). Die gegenwärtige Informationslage erlaubt keine eindeutige Entscheidung für eine dieser Anpassungen. Anknüpfend an die von MüLlER \& SuEss (1979) gegebene Beziehung zwischen den beiden hier diskutierten Parametern soll in der weiteren Diskussion auf den Zusammenhang zurückgegriffen werden, der durch die Anpassungskurve (2) in Abb. 7 dargestellt wird.

Der hier beschriebene Zusammenhang für die Sedimente des Großen Jasmunder Boddens stimmt damit nicht mit der Auffassung von MüLler \& SuEss (1979) für marine Sedimente überein, daß zwischen Sedimentationsraten und Akkumulationsraten organischen Kohlenstoffs eine Potenzfunktion in der Form

also

$$
\begin{aligned}
& 2 \mathrm{C}_{\text {org }}-\mathrm{AR}=(1,6 \cdot \mathrm{a} \cdot \mathrm{SR})^{\mathrm{b}}, \\
& \mathrm{C}_{\text {org }}-\mathrm{AR}=(\mathrm{a} \cdot \mathrm{SR})^{1,47}
\end{aligned}
$$

besteht. MüLLER \& SuEss (1979) ermittelten in ihrer Studie einen Exponenten $>1$, woraus sich ein konvexer Verlauf der Anpassungskurve ergibt, während in den hier gezeigten Funktionen jeweils ein Exponent $<1$ auftritt, der einen konkaven Verlauf der Kurve bewirkt ${ }^{2}$. Dabei weisen die Autoren zwar darauf hin, daß bei den von ihnen vermuteten Zusammenhängen Veränderungen in der Produktivität nicht berücksichtigt werden, die im ozeanischen Bereich bis zu einem gewissen Grad mit den Veränderungen in den Sedimentationsraten korrespondieren. Das hat zur Folge, daß der Grad der Erhaltung von organischer Substanz als Folge erhöhter Sedimentationsraten überschätzt wird. Der Unterschied zwischen dem Exponenten von 0,25 in den Sedimenten des Großen Jasmunder Boddens und dem Exponenten 1,47 von MüL-

2) Der Vergleich mit der zweidimensionalen Gleichung vOn MÜLLER \& Suess (1979) wird möglich, wenn in der in Abb. 7 gegebenen Gleichung die Sedimentakkumulationsrate in die Faktoren Sedimentationsrate und Dichte zerlegt und, wie bei MülLER \& SuESS (1979), eine konstante Dichte der Sedimente angenommen wird. 
LER \& Suess (1979) erscheint aber zu groß, um die von den Autoren beschriebene Überschätzung der $\mathrm{C}_{\text {org }}$-Akkumulationsraten allein widerzuspiegeln. Vielmehr ist für die Sedimente des Großen Jasmunder Boddens denkbar, daß der Zusammenhang zwischen erhöhter Produktivität und daraus resultierenden erhöhten Sedimentationsraten nicht so vordergründig in Erscheinung tritt, wie im ozeanischen Bereich. Ein Grund dafür kann sein, daß der Große Jasmunder Bodden sehr flach ist und durch eine entsprechend gute Wasserdurchmischung und gute Sauerstoffversorgung sowie ständiges Auftreten von Resuspensions- und Resedimentationsprozessen gekennzeichnet ist. Das Gewässer bietet Mikroorganismen damit gute „Angriffsmöglichkeiten" für den biologischen Abbau, weil die organische Substanz auf diese Weise trotz relativ hoher Sedimentationsraten nicht ,versiegelt" wird (Emerson \& Hedges 1988). Um der Tatsache Rechnung zu tragen, daß die $\mathrm{C}_{\text {org }}$-Akkumulationsraten erst ab einem bestimmten Wert der Sedimentakkumulationsraten anfangen zuzunehmen, wurde zur Korrektur ein zusätzlicher Summand in die Gleichungen einbezogen.

\subsection{Biogene Komponenten}

Die biogene Komponente von Meeres- und Boddensedimenten besteht neben organischen Verbindungen hauptsächlich aus zwei Substanzen, die als Hartteile von Organismen produziert werden. Diese sind Kalzit und Opal, die durch das im Wasser lebende Plankton ausgefällt werden. Dabei reflektiert das Vorkommen dieser Stoffe am Gewässerboden, wie von Broecker \& PENG (1982) für den Ozean nachgewiesen, sowohl die Produktionsrate in der darüberliegenden Wassersäule als auch den Umfang, in dem diese Stoffe der Lösung ausgesetzt sind.

Meerwasser ist mit Hinblick auf Kieselsäure in der Regel immer untersättigt (BRoECKer \& PeNG 1982). Das trifft auch für die Bodden und Haffe zu, wodurch es zu Auflösungserscheinungen kommt, wie sie auch an Diatomeen in den Boddensedimenten beobachtet werden können (JANKE, pers. Mitt.). Damit ist eine Fehlinterpretation der Resultate denkbar, wenn diese als Paläoproduktivitätsindikatoren angewendet werden. Dennoch wird in vielen Arbeiten davon ausgegangen, daß Perioden erhöhter Produktivität mit Perioden erhöhter Einlagerung von biogenem Opal in das Sediment einhergehen, weil Produktion, Auflösung und Einlagerung im gleichen Verhältnis zueinander erfolgen (BROECKER \& PENG 1982). Die Auflösung von Karbonatschalen in den Sedimenten wird für die Bodden und Haffe am Beispiel des Greifswalder Boddens ausführlich in FrenzeL (1996) diskutiert.

Die Gehalte an organischem Kohlenstoff liegen in den Sedimenten des Großen Jasmunder Boddens in der Größenordnung von 4-8\% im Jüngeren und Älteren Atlantikum und von 2-4\% im Subboreal. Eine deutliche Erhöhung der $C_{\text {org }}$-Gehalte kann mit dem Einsetzen des Subatlantikums beobachtet werden. Zu Beginn dieser Pollenzone liegen die Gehalte um 2-3\%. Die Werte nehmen bis in die Gegenwart hin zu, wobei die erkennbar höchsten Gehalte in den letzten 200 Jahren auftreten. In den Oberflächensedimenten wurden Werte um 10-11\% gemessen.

Relativ hohe Opalgehalte um 2-5\% liegen im Älteren und in der ersten Hälfte des Jüngeren Atlantikums vor. In der zweiten Hälfte des Jüngeren Atlantikums liegen sie zumeist um 1-2\%. Ein deutliches Optimum tritt im Subboreal von 4500 BP bis $3500 \mathrm{BP}$ mit Werten $>4 \%$ und einem Maximum von $7 \%$ auf. In der zweiten Hälfte des Subatlantikums kommt es zu einem allgemeinen Anstieg der Opalanteile auf zumeist $>2 \%$ mit Werten bis etwa $6 \%$. Sowohl die Zunahme der $\mathrm{C}_{\text {org }}$-Gehalte als auch die Erhöhung der Opalwerte könnten auf Eutrophierungstendenzen im Großen Jasmunder Bodden zurückzuführen sein.

Die $\mathrm{CaCO}_{3}$-Gehalte in den Sedimenten des Großen Jasmunder Boddens liegen im Älteren Atlantikum unter $10 \%$. Relativ hohe $\mathrm{CaCO}_{3}$-Gehalte von über $10 \%$ finden sich im Jüngeren Atlantikum. Im Subboreal, im Älteren Subatlantikum und teilweise im Mittleren Subatlantikum liegen die $\mathrm{CaCO}_{3}$-Gehalte häufig über $5 \%$, und es treten bis etwa 900 Einzelwerte von über $20 \%$ auf, die auf das Vorkommen von Schillagen zurückgeführt werden können. Nach 900 BP kommen diese nicht mehr vor. Eine Zunahme der $\mathrm{CaCO}_{3}-$ Werte zur Gegenwart hin, wie sie für die $\mathrm{C}_{\text {org }}$ - und Opalgehalte beobachtet wurde, ist nicht festzustellen. Die Werte legen nahe, daß autochthone karbonatbildende Organismen im Großen Jasmunder Bodden von geringer Bedeutung für den Kalziumkarbonateintrag in das Sediment sind. Produktivitätsschwankungen sind nicht nachweisbar, weil der Anteil des von karbonatbildenden Organismen stammenden Kalziumkarbonats gegenüber Einträgen von der Erosion her vernachlässigbar gering ist. In der Umgebung des Großen Jasmunder Boddens befinden sich viele größere eiszeitliche Senken und Geschiebemergelhänge. Unter dem Geschiebemergel liegen in der Regel schlecht sortierte grobe Sande, die 

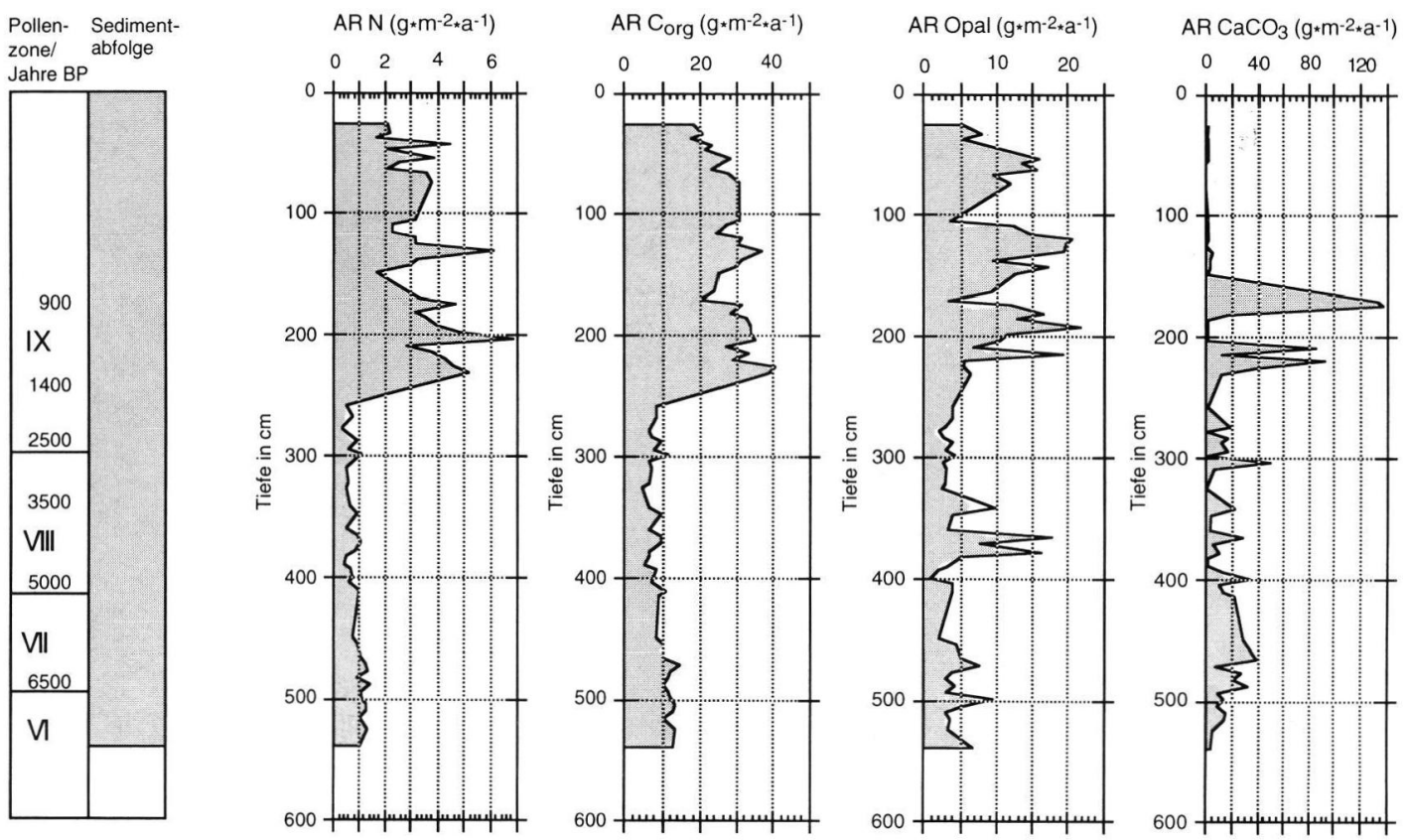

Abb. 8: Akkumulationsraten für die Sedimente aus dem Großen Jasmunder Bodden (Kern GJB1): Stickstoff, organischer Kohlenstoff, biogener Opal und Kalziumkarbonat (jweils in $\mathrm{g} \cdot \mathrm{m}^{-2} \cdot \mathrm{yr}^{-1}$ ). Links: Pollenzonen mit Altersangaben BP und Sedimentabfolge (Schlick).

Fig. 8: Accumulation rates for the Großer Jasmunder Bodden sediments (core GJB1): nitrogen, organic carbon, biogenic opal and calcium carbonate (all in $\mathrm{g} \cdot \mathrm{m}^{-2} \cdot \mathrm{yr}^{-1}$ ). The three left columns show years BP, pollen zones and the sediment sequence (mud).

ebenfalls Kalke enthalten. Auch die Bedeutung von Schillagen für den Kalziumkarbonatgehalt im Sediment darf für den Großen Jasmunder Bodden nicht vernachlässigt werden. Ungefähr zeitgleich mit der vollständigen Abschließung des Großen Jasmunder Boddens von der Ostsee, die gegenwärtig existierenden Auffassungen zufolge wahrscheinlich erst im Subatlantikum erfolgte (KLIBO 1995), tritt ein Rückgang der $\mathrm{CaCO}_{3}$-Anteile im Sediment auf. Es liegt zudem nahe, daß die mit der Abriegelung des Boddens verbundenen veränderten Umweltbedingungen in dem Gewässer, einschließlich der zunehmenden Aussüßung, einen Rückgang der Produktivität der vorhandenen karbonatbildenden Organismen bedeuteten. Vor der Abriegelung waren aufgrund des Buchtcharakters und einer gewissen Salinität des Gewässers sehr gute Lebensbedingungen für Organismen gegeben (JANKE, pers. Mitt.).

\subsection{Akkumulationsraten einzelner Komponenten}

Die Akkumulationsraten (AR) einzelner Komponenten des Sediments, Maß für die Trockenmasse von Material, das pro Flächen- und Zeiteinheit abgelagert wird, erlauben den Vergleich der tatsäch- lichen Ablagerungsraten in Sedimenten mit variierendem Wassergehalt (REA et al. 1980). Die Akkumulationsraten organischen Kohlenstoffs können z. B. Auskunft geben über Einträge organischer Substanz, die Höhe der Produktivität eines Gewässers, aber auch über die Intensität von Abbauprozessen organischer Substanz als Funktion anderer Milieuparameter. Aufgrund der Vielzahl von möglichen Quellen organischen Materials sind Akkumulationsraten von organischem Kohlenstoff für den Großen Jasmunder Bodden nur eingeschränkt als Produktivitätsindikator anwendbar.

Die $\mathrm{C}_{\text {org }}$-Akkumulationsraten im Großen Jasmunder Bodden (Abb. 8) streuen zwischen 4,5 $\mathrm{g} \cdot \mathrm{m}^{-2} \cdot \mathrm{a}^{-1}$ und $40,7 \mathrm{~g} \cdot \mathrm{m}^{-2} \cdot \mathrm{a}^{-1}$. Sie liegen im erfaßten Teil des Jüngeren Atlantikums und über das gesamte Ältere Atlantikum hinweg um $10 \mathrm{~g} \cdot \mathrm{m}^{-2} \cdot \mathrm{a}^{-1}$. Danach sind sie bis zum Subatlantikum immer niedriger als $10 \mathrm{~g} \cdot \mathrm{m}^{-2} \cdot \mathrm{a}^{-1}$. Die höchsten $\mathrm{C}_{\text {org }}$-Akkumulationsraten treten im Subatlantikum ab ca. 1400 BP bis zur Gegenwart hin auf, wobei überwiegend Werte zwischen 20 und $30 \mathrm{~g} \cdot \mathrm{m}^{-2} \cdot \mathrm{a}^{-1}$, mit Maximalraten bis $40 \mathrm{~g} \cdot \mathrm{m}^{-2} \cdot \mathrm{a}^{-1}$ vorliegen. 
Im Großen Jasmunder Bodden zeigen die Akkumu-lationsraten für Sticktoff (Abb. 8) die gleichen Trends wie die Akkumulationsraten für organischen Kohlenstoff. Die Werte vom erfaßten Teil des Älteren Atlantikums bis in das Subatlantikum hinein (etwa 1900/1800 BP) liegen um $1 \mathrm{~g} \cdot \mathrm{m}^{-2} \cdot \mathrm{a}^{-1}$. Dabei sind die Werte im Jüngeren und Älteren Atlantikum zumeist geringfügig höher. Ab 1400 BP kam es zu einer Ablagerung von $>2 \mathrm{~g} \cdot \mathrm{m}^{-2} \cdot \mathrm{a}^{-1}$, wobei einzelne Werte zwischen 1400 und 900 BP mit $>5 \mathrm{~g} \cdot \mathrm{m}^{-2} \cdot \mathrm{a}^{-1}$ geringfügig höher sind als in den letzten 900 Jahren vor der Gegenwart. Für die letzten 150 Jahre vor der Gegenwart liegen keine Werte vor.

Die Akkumulationsraten für Opal (Abb. 8) streuen im Großen Jasmunder Bodden von 0,8 bis $21,8 \mathrm{~g} \cdot \mathrm{m}^{-2} \cdot \mathrm{a}^{-1}$. Im Atlantikum, Subboreal und Subatlantikum liegen bis etwa 1400 BP zumeist Werte $<5 \mathrm{~g} \cdot \mathrm{m}^{-2} \cdot \mathrm{a}^{-1}$ vor. Hohe Opal-Akkumulationsraten von $>10 \mathrm{~g} \cdot \mathrm{m}^{-2} \cdot \mathrm{a}^{-1}$ treten vereinzelt im Subboreal auf. Zudem kommt es ab ca. 2200 BP zu einem leicht zunehmenden Trend der Werte. Ein deutliches Ansteigen der Opal-Akkumulationsrate, mit einer Ablagerung von 10 bis $20 \mathrm{~g} \cdot \mathrm{m}^{-2} \cdot \mathrm{a}^{-1}$, läßt sich ab ca. 1300 BP nachweisen. Dabei hat es den Anschein, daß dieser mittlere und jüngere $A b-$ schnitt des Subatlantikums hinsichlich der Ablagerungsrate von Opal in drei Zeitabschnitte untergliedert werden kann, in denen es jeweils zu einem Optimum in der Produktivität opalbildender Organismen kam. Demzufolge erfolgte der erste Anstieg der Produktivität ab 1300 BP mit Werten bis über $20 \mathrm{~g} \cdot \mathrm{m}^{-2} \cdot \mathrm{a}^{-1}$, die dann bis ca. 1050 auf Werte um $7 \mathrm{~g} \cdot \mathrm{m}^{-2} \cdot \mathrm{a}^{-1}$ zurückgingen. Danach kam es zu einer erneuten Zunahme der Einlagerung opalbildender Organismen mit erneuten Maxima um $20 \mathrm{~g} \cdot \mathrm{m}^{-2} \cdot \mathrm{a}^{-1}$. Ein weiteres Produktivitätsminimum mit Werten unter $5 \mathrm{~g} \cdot \mathrm{m}^{-2} \cdot \mathrm{a}^{-1}$ tritt um 500 BP auf. Von 500 bis 200 BP liegt dann das 3. Intervall einer hohen, bis auf ca. $15 \mathrm{~g} \cdot \mathrm{m}^{-2} \cdot \mathrm{a}^{-1}$ ansteigenden Opal-Akkumulationsrate vor, die um $200 \mathrm{BP}$ erneut auf Werte um $6 \mathrm{~g} \cdot \mathrm{m}^{-2} \cdot \mathrm{a}^{-1}$ abfällt. Für die letzten 200 Jahre liegen keine Opal-Akkumulationsraten vor.

Die $\mathrm{CaCO}_{3}$-Akkumulationsraten für Kern GJB1 liegen im Bereich von 0,1 bis $136,8 \mathrm{~g} \cdot \mathrm{m}^{-2} \cdot \mathrm{a}^{-1}$. Die Mehrzahl der Datenwerte fällt unter $20 \mathrm{~g} \cdot \mathrm{m}^{-2} \cdot \mathrm{a}^{-1}$, wobei ab ca. $750 \mathrm{BP}$ deutlich niedrigere Werte vorliegen. Die $\mathrm{CaCO}_{3}$-Akumulationsraten sind im Jüngeren Atlantikum $\left(>20 \mathrm{~g} \cdot \mathrm{m}^{-2} \cdot \mathrm{a}^{-1}\right)$ und $\mathrm{zu}$ Beginn des Subatlantikums $\left(>15 \mathrm{~g} \cdot \mathrm{m}^{-2} \cdot \mathrm{a}^{-1}\right)$ recht hoch. Ab ca. $1400 \mathrm{BP}$ treten die bereits für die Gewichtsanteile erwähnten Spitzenwerte einzelner
Proben über mehrere Jahrhunderte hinweg auf (Abb. 8). Nach 800 BP sind die $\mathrm{CaCO}_{3}$-Akumulationsraten erneut sehr gering, überwiegend unter $1 \mathrm{~g} \cdot \mathrm{m}^{-2} \cdot \mathrm{a}^{-1}$. Nur Einzelwerte an der Oberfläche erreichen 5 bis $6 \mathrm{~g} \cdot \mathrm{m}^{-2} \cdot \mathrm{a}^{-1}$. Ein zur Gegenwart hin ansteigender Trend konnte für die $\mathrm{CaCO}_{3}-\mathrm{Ak}$ kumulationsraten nicht beobachtet werden. Das unterstützt die Aussage, daß autochthone Einträge von Kalziumkarbonat vernachlässigbar gering sind gegenüber allochthonen Einträgen.

\section{Danksagung}

Herrn Prof. Dr. W. Janke möchte ich für die Durchführung der Pollenanalysen sowie für wertvolle Hinweise zur Diatomeenflora des Großen Jasmunder Boddens danken. Diese Arbeit wurde vom Bundesministerium für Bildung, Wissenschaft, Forschung und Technologie (BBWFT Project 01 LK 9405/4 KLIBO) unterstützt.

\section{Schriftenverzeichnis}

BERnER, R. A. (1970): Sedimentary pyrite formation. American Journal of Science, 268: 1-23; New Haven/Conn.

BERNER, R. A. \& RAISWELL, R. (1983): Burial of organic carbon and pyrite sulfur in sediments over Phanerozoic time: a new theory. - Geochimica et Cosmochimica Acta, 47: 855-862; Oxford.

BirR, H.-D. \& OERTEL, P. (1993): About the dynamics of Baltic Sea water inflow into the Bodden waters around Rügen. - Petermanns Geogr. Mitt., 137: 283 288; Gotha.

BoRDOVSKIY, O. K. (1965): Accumulation and transformation of organic substances in marine sediments. - Marine Geology, 3: 3-114; Amsterdam.

Broecker, W. S.; Peng, T. H. (1982): Tracers in the Sea: 690 S.; New York (Eldigio Press).

ConRad, K.; Bartels S.; Eidam, J.; JÖnsSOn, N.; KlÜBer, U.; Luther, T.; MeYer, H.; Miske, V.; Suchau, A.; VietingHOFF, U. \& WINTER, M. (1995): Erfassung des Bewuchses des Greifswalder Boddens mit submersen Makrophyten und der Akkumulation von C, N, P, S, $\mathrm{Mg}$ und Spurenelementen durch Potamogeton pectinatus und andere Arten. - GOAP 2. Statusseminar Greifswald: 35-36; Greifswald.

CORRENS, M. (1976): Charakteristische morphometrische Daten der Bodden und Haffgewässer der DDR. Vermessungstechnik, 24: 459-461.

Correns, M. \& JaeGer, F. (1979): Beiträge zur Hydrographie der Nordrügenschen Bodden. I. Einführung in das Untersuchungsgebiet, Wasserstandsverhältnisse und Wasserhaushalt. - Acta hydrophys., 24 (3) 149-177.

DAHLKE, S. \& HÜBEL, H. (1994): Stoff- und Energiertransfer in den flachen Küstengewässern der deutschen Ost- und Nordsee und Entwicklung von dazu- 
gehörenden Meßtechnologien und Systemen (Rügensche Boddengewässer/SWH-Wattenmeer) - SEFON. Forschungsbericht, Universität Greifswald/ Hiddensee, 77 S.; Kloster/Hiddensee.

DeMASTER (1981): The supply and accumulation of sili$\mathrm{ca}$ in the marine environment. - Geochimica et Cosmochimica Acta, 45: 1715-1732; Oxford.

EmeIS, K.-C. \& Morse, J.W. (1993): Zur Systematik der Kohlenstoff-Schwefel-Eisen-Verhältnisse in Auftriebssedimenten. - Geologische Rundschau, 82: 604-618; Stuttgart

Emerson, S. \& Hedges, J. I. (1988): Processes controlling the organic carbon content of open ocean sediments. - Paleoceanography, 3: 621-634; Cambridge/ Mass.

Faure, G. (1986): Principles of Isotope Geology (2nd edition): 589 S.; New York (Wiley)

Fontugne, M. R. \& Jouanneau, J. M. (1987): Modulation of the particulate organic carbon flux to the ocean by a macrotidal estuary - evidence from measurements of carbon isotopes in organic matter from the Gironde system. - Estuarine, Coastal and Shelf Science, 24 (3): 377-387; London.

Frenzel, P. (1996): Rezente Faunenverteilung in den Oberflächensedimenten des Greifswalder Boddens (südliche Ostsee) unter besonderer Berücksichtigung der Ostrakoden (Crustacea). - Senckenbergiana maritima, 27 (1/2): 11-31; Frankfurt a. M.

Galimov, E.M. (1985): The Biological Fractionation of Isotopes: $261 \mathrm{~S}$; Orlando (Academic Press).

Hebbeln, D. (1991): Spätquartäre Stratigraphie und Paläozeanographie in der Fram-Straße. - Berichte FB Geowissenschaften Univ. Bremen, 22: 174 S.; Bremen.

KLIBO-Jahresbericht (1995): Klimawirkung und Boddenlandschaft. - BBWFT-Projekt 01 LK 9405/4: 55 S.; Greifswald.

Kliewe, H. (1987): Zur Morphogenese der Bodden und ihrer Randniederungen. - Wiss.Zeitschr. Univ. Greifswald, Math.-nat.wiss.Reihe, 36 (2-3): 94-98; Greifswald.

KLIEWE, H. \& JANKE, W. (1978): Zur Stratigraphie und Entwicklung des nordöstlichen Küstenraumes der DDR. - Petermanns Geogr. Mitt., 122: 81-91; Gotha.

- (1982): Der holozäne Waserspiegelanstieg der Ostsee im nordöstlichen Küstengebiet der DDR. - Petermanns Geogr. Mitt., 135: 1-14; Gotha.

- (1991): - Holozäner Küstenausgleich im südlichen Ostseegebiet bei besonderer Berücksichtigung der Boddenausgleichsküste Vorpommerns. - Petermanns Geogr. Mitt., 1: 1-14; Gotha.

LANGe, E., Jeschke, L. \& Knapp, H. D. (1986): Ralswiek und Rügen. Landschaftsentwicklung und Siedlungsgeschichte der Ostseeinsel. Teil 1. Die Landschaftsgeschichte der Insel Rügen seit dem Spätglazial. Text und Beilagen. - Schriften zur Ur- und Frühgeschichte, 38, $175 \mathrm{~S}$.

Leipe, T., Neumann, T. \& EmeIs, K.-C. (1995): Schwermetallverteilung in holozänen Ostseesedimenten. Geowissenschaften, 13 (12): 470-478.

MARIOTTI, A. (1983): Atmospheric nitrogen is a reliable standard for natural $15 \mathrm{~N}$ abundance measurements. - Nature, 303: 680-683; London.
Mortlock, R.D. \& Froelich, M. A. (1989): A simple method for the rapid determination of biogenic opal in pelagic marine sediments. - Deep-Sea Research, 36: 1415-1426; Oxford.

Müller, A. (1997): Zu Sedimentationsgeschichte und Paläomilieu der Darß-Zingster Boddenkette (Barther Bodden und Grabow), südliche Ostsee. - Zeitschrift für Geologische Wissenschaften (eingereicht)

Müller, A., Janke, W. \& Lampe, R. (1996): Zur Sedimentationsgeschichte des Oderhaffs. - Bodden, 3: 167172; Kloster/Hiddensee.

Müller, A. \& Mathesius, U. (1997): The application of sedimentary $\mathrm{C}_{\text {org }} / \mathrm{N}$ and $13 \mathrm{C} / 12 \mathrm{C}$ ratios as source indicators of organic matter in coastal lagoons of the southern Baltic Sea. - Palaeogeography, Palaeoclimatology, Palaeoecology (im Druck).

Müller, A. \& Voss, M. (1997): The paleoenvironment of coastal lagoons in the southern Baltic Sea as derived from $\delta^{13} \mathrm{C}$ and $\delta^{15} \mathrm{~N}$ ratios. - Palaeogeography, Palaeoclimatology, Palaeoecology (im Druck).

MüLlER, P. J. (1975): Diagenese stickstoffhaltiger organischer Substanzen in oxischen und anoxischen marinen Sedimenten. - Meteor Forsch.-Ergebn., Reihe C, 22: 1-60; Berlin.

Müller, P. J. \& SuEss, E. (1979): Productivity, sedimentation rate and organic matter in the oceans. - I. Organic carbon preservation. - Deep-Sea Research, 26: $1347-1362$; Oxford.

Nakai, N. (1986): Paleoenvironmental features of Lake Biwa deduced from carbon isotope compositions and organic $\mathrm{C} / \mathrm{N}$ ratios of the upper $800 \mathrm{~m}$ sample of 1,400 cored column. Proceedings of the Japan Academy 62B (8): 279-282; Tokyo.

Neumann, T., Leipe, T., Brand, T. \& Shimmield, G. (1996): Accumulation of heavy metals in the Oder Estuary and its off-shore basins. - Chemie der Erde, 56: 207 222; Jena.

Owens, N.J.P. (1987): Natural variations in $15 \mathrm{~N}$ in the marine environment. - Advances in Marine Biology, 24: 411-451; London.

Peters, K. E., Sweeney, R. E. \& Kaplan, I. R. (1978): Correlation of carbon and nitrogen stable isotope ratios in sedimentary organic matter. - Limnology and Oceanography, 23: 598-604; Lawrence/Kansas.

Prahl, F. G. \& Bennett, J. T. \& Carpenter, R. (1980): The early diagenesis of aliphatic hydrocarbons and organic matter in sedimentary particulates from Dabob Bay, Washington. - Geochimica et Cosmochimica Acta, 44: 1967-1976; Oxford.

ReA, D. K.; Bourbonniere, R. A. \& Meyers, P. A. (1980): Southern Lake Michigan sediments: Changes in accumulation rate, mineralogy, and organic content. Journal Great Lakes Research, 6: 321-330.

RuttenberG, K. C. \& Goñi, M. A. (1997): Phosphorus distribution, C:N:P ratios, and $\delta 13 \mathrm{C}_{\mathrm{oC}}$ in arctic, temperate, and tropical coastal sediments: tools for characterizing bulk sedimentary organic matter. Marine Geology, 139: 123-145; Amsterdam.

Schoeninger, M. J. \& DeNiro, M. J. (1984): Nitrogen and carbon isotope composition of bone collagen from marine and terrestrial animals. - Geochimica et Cosmochimica Acta, 48: 625-639; Oxford. 
StraHt, J. (1996): Pollenanalytische Untersuchung eines Vibrokernprofils aus dem NW-Teil des Greifswalder Boddens, südliche Ostsee. - Senckenbergiana maritima, 27 (1/2): 49-56; Frankfurt a.M.

SWEenEY, R. E. \& KAPLAN, I.R. (1980): Natural abundance of $15 \mathrm{~N}$ as a source indicator for near-shore marine sedimentary and dissolved nitrogen. - Marine Chemistry, 9: 81-94; Amsterdam.

Thiede, J.; Suess, E. \& Müller, P. J. (1982): Late Quarternary fluxes of major sediment components to the sea floor at the Northwest African continental slope. In: Von RAD, U.; Hinz, K.; SARnTHeim, M. \& Seibold, E. (eds.): Geology of the Northwest African Continental Margin: 605-631; Berlin etc. (Springer).

Thornton, S. F. \& McManus, J. (1994): Application of organic carbon and nitrogen stable isotope and $\mathrm{C} / \mathrm{N}$ ratios as source indicators of organic matter provenance in estuarine systems: Evidence from the Tay Estuary, Scotland. - Estuarine, Coastal and Shelf Science, 38: 219-233; London.
TOMCZAK M. \& Godfrey, J.S. (1994): Regional oceanography: an introduction: 422 S.; London (Pergamon).

Van Andel, T. H.; Heath, G. R. \& Moore, T. C. (1975): Cenozoic history and paleoceanography of the central equatorial Pacific Ocean. - Geol.Soc.Am.Mem., 143: 134 S.; New York.

Voss, M. \& STRUCK, U. (1997): Stable nitrogen and carbon isotopes as indicator of eutrophication of the Oder River (Baltic Sea). - Marine Chemistry, 59: 3549; Amsterdam.

Manuskript eingegangen am 10. Februar 1998 